\title{
COVID-19 comorbidity and metabolic syndrome: is there a Molecular basis?
}

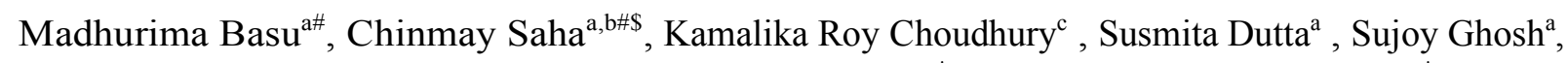
Subhankar Chowdhury ${ }^{\mathrm{a}}$, Satinath Mukhopadhyay ${ }^{\mathrm{a}^{*}}$, and Nitai P. Bhattacharyya $\mathrm{a}^{\mathrm{a}^{*}}$

\footnotetext{
${ }^{a}$ Department of Endocrinology and Metabolism, Institute of Post Graduate Medical Education \& Research and Seth Sukhlal Karnani Memorial Hospital, Kolkata 700020, West Bengal, India.

${ }^{\mathrm{b}}$ Department of Genome Science, School of Interdisciplinary Studies, University of Kalyani, Kalyani, Nadia 741235, West Bengal, India.

${ }^{\mathrm{c}}$ Cell Biology \& Physiology Division, CSIR-IICB (TRUE campus), Kolkata-700091, India
}

${ }^{\#}$ These authors contributed equally to this work

${ }^{\$}$ Current address: Department of Genome Science, School of Interdisciplinary Studies, University of Kalyani, Kalyani, Nadia 741235, West Bengal, India.

\section{*Correspondence should be addressed either to}

Nitai P. Bhattacharyya, Ph.D, Department of Endocrinology and Metabolism, Institute of Post Graduate Medical Education \& Research and Seth Sukhlal Karnani Memorial Hospital, Kolkata 700020, West Bengal, India, Email Id: nitai.bhattacharyya@gmail.com

or

Satinath Mukhopadhyay, DM, FRCP (London), Department of Endocrinology and Metabolism, Institute of Post Graduate Medical Education \& Research and Seth Sukhlal Karnani Memorial Hospital, Kolkata 700020, West Bengal, India, email Id: satinath.mukhopadhyay@gmail.com

\begin{abstract}
The risk factors associated with COVID-19 related severity, morbidity, and mortality, i.e., obesity (often associated with NAFLD), hyperglycemia, hypertension and dyslipidemia all cluster together as metabolic syndrome (MetS). Instead of studying association of these risk factors with COVID-19, it makes sense studying the association between MetS on one hand and COVID-19 on the other. This study explores a molecular basis underpinning the above association. Severity of COVID-19 patients with MetS could be due to functional alterations of host proteins due to their interactions with viral proteins. We collected data from Enrichr (https://amp.pharm.mssm.edu/Enrichr/), DisGeNET (https://www.disgenet.org/) and others and carried out enrichment analysis using Enrichr. Various biological processes and pathways associated with viral protein interacting partners are known to involve in metabolic diseases. The molecular pathways underlying insulin resistance, insulin signaling and insulin secretion are not only involved in diabetes but also in CVD and obesity (associated with
\end{abstract}


non-alcoholic fatty liver disease; NAFLD). Lipid metabolism/lipogenesis, fatty acid oxidation and inflammation are associated with MetS. Viral interacting host proteins are associated and enriched with terms like hyperglycemia, coronary artery disease, hypertensive disease related to CVD and liver diseases in DisGeNET. Association of viral interacting proteins with disease-relevant biological processes, pathways and disease-related terms suggests that altered host protein function following interaction with viral proteins might contribute to frequent occurrence and/or severity of COVID-19 in subjects with MetS. Such analysis not only provides a molecular basis of comorbidity but also incriminates host proteins in viral replication, growth and identifies possible drug targets for intervention.

\section{Keywords}

COVID-19, Metabolic syndrome, Comorbidity, Enrichment analysis, biological processes and pathways 


\section{Graphical Abstract}

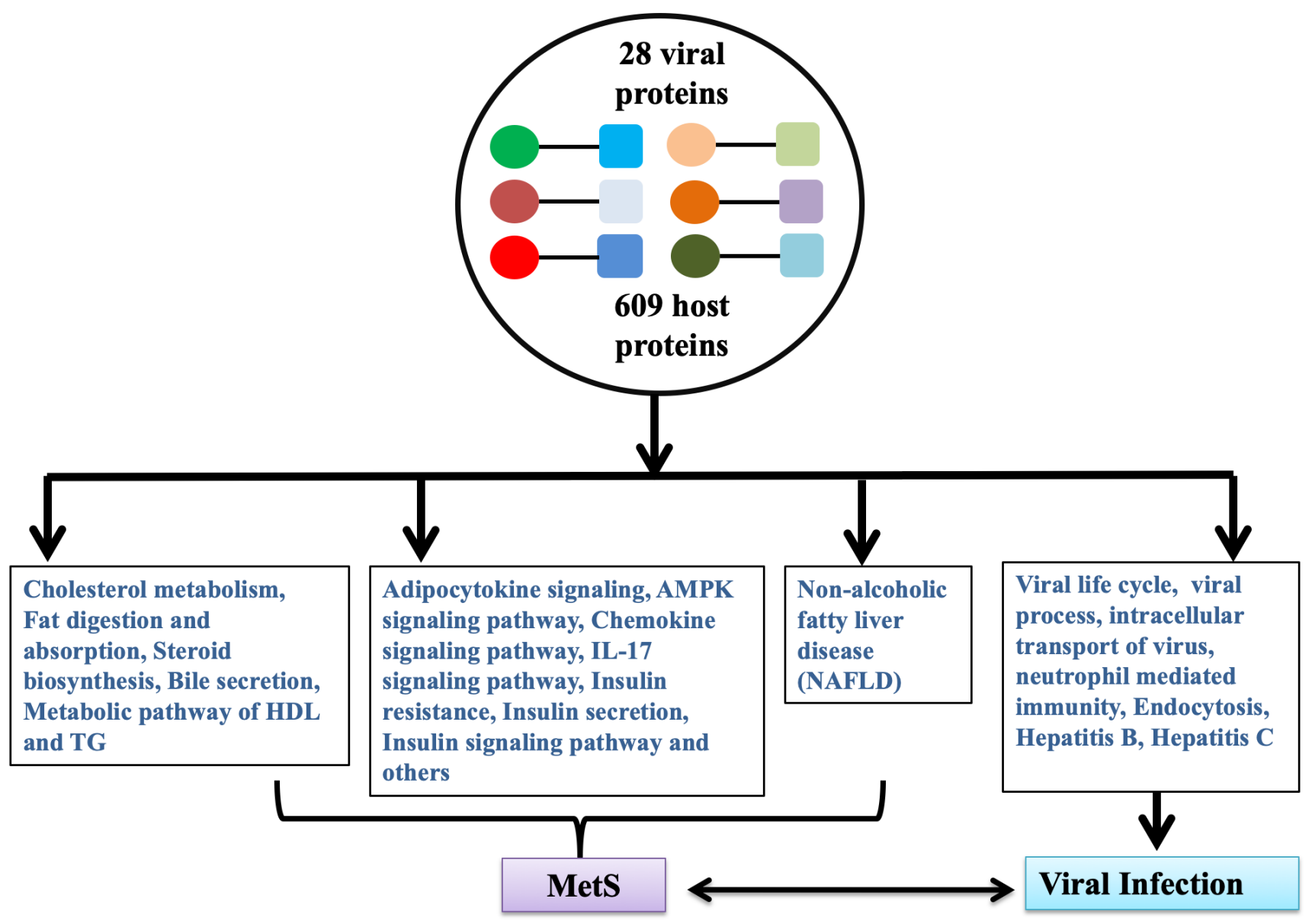

\section{Highlights}

- Viral protein interacting host proteins are enriched with diverse biological processes (BPs) and pathways

- These interacting host proteins are also enriched with disease/trait in DisGeNET

- Enriched BPs and pathways are relevant for viral growth and survival

- Enriched BPs, pathways and diseases are relevant for MetS

- Functional changes of host proteins in infection and MetS might explain the severity of COVID-19 


\section{Introduction}

Coronavirus disease 19 (COVID-19) is caused by the infection with severe acute respiratory syndrome corona virus 2 (SARS-CoV-2). Starting in the last quarter of 2019 in the Wuhan City of China, it spread rapidly to affect populations in almost all countries worldwide [8061550 people infected worldwide and causing death of 440290 (WHO Situation report 149, 17 th June 2020)]. Comorbid conditions like hypertension, acute myocardial infarction, obesity, liver and kidney injuries, cardiovascular disease (CVD), type 2 diabetes (T2D), chronic obstructive pulmonary disease (COPD) and gastrointestinal diseases are over represented among COVID-19 patients. In severe cases, patients quickly progress to acute respiratory distress syndrome (ARDS), septic shock, metabolic acidosis, and coagulopathy. Studies have shown that older ( $>60$ years) COVID-19 patients with hypertension, diabetes, COPD, CVD, liver and gastrointestinal disease and kidney disease to be susceptible to severe and rarely fatal outcomes [1-10]. Result presented in these publications showed that (i) age (ii) gender and (iii) comorbidity are risk factors for severity and/ or death.

\subsection{Metabolic syndrome and comorbidity of COVID-19}

Metabolic syndrome (MetS) consists of a cluster of inherited metabolic traits that center on obesity, insulin resistance, hypertension and atherogenic dyslipidemia and contribute to the prevalence of CVD, T2D, NAFLD and cancer (Figure 1). These traits were more prevalent among people with severe COVID-19 compared to their non-severe counterparts [3, 6, 11, 12]. Detailed information of the co-existence of obesity, CVD, hypertension, diabetes and NAFLD is shown in the supplementary Text (Supplementary text 1.1). It is thus pertinent to consider whether metabolic syndrome may be considered as co-morbid condition for COVID-19.

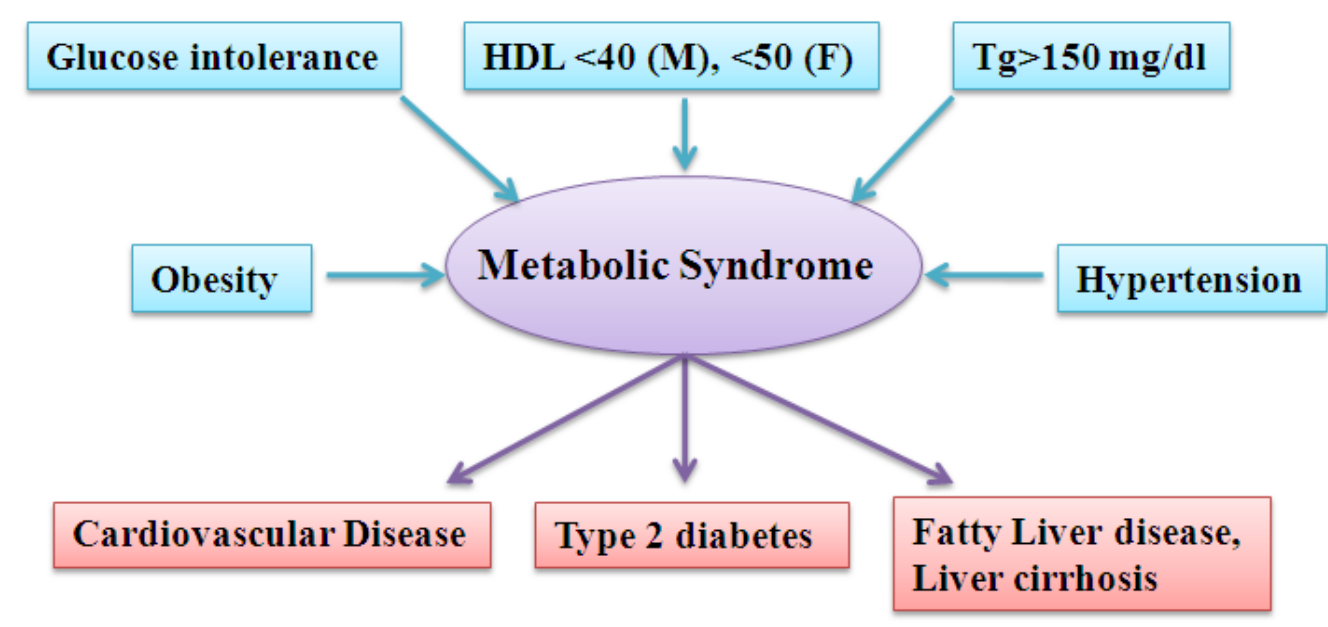


Figure 1 Components of metabolic syndrome and long term outcome of metabolic syndrome ([13] [14]). HDL: high-density lipoprotein, Tg: triglyceride.

\subsection{Altered expression of host genes in virus infected cells}

Altered expression of host genes in vitro following infection with SARS-CoV-2 has been observed $[15,16]$. Analysis of host transcriptional response to SARS-CoV-2 and other respiratory infections through in vitro, ex vivo and in vivo model systems reveal that infection deregulates many genes that serve as unique transcriptional footprints for the virus. Comparison with influenza A and respiratory syncytial viruses reveals an altered response that lacks robust induction of cytokine and chemokine. Transcriptional profiling in lung biopsy specimens from COVID-19 subjects reveal a unique and inappropriate inflammatory response defined by elevated chemokine expression [16]. Altered expressions of host genes on infection with many viruses including SARS-CoV are catalogued in Enrichr at https://amp.pharm.mssm.edu/Enrichr/ [17, 18].

\subsection{Interactions between host proteins and SARS-CoV-2 coded viral proteins}

Viral proteins, including SARS-CoV and other human corona virus coded proteins interact with host proteins [1, 19-21]. Such interactions hijack host proteins and modify cellular processes and pathways to evade immune response of the host and survival of viruses. Interaction of viral protein with the host proteins might modulate the functions of the host proteins by (i) changing cellular localization, transport and interaction with other host proteins, (ii) changing activation of the host proteins by post translational modifications and (iii) degradation of the host proteins. SARS-CoV coded ORF6 interacts with STAT1 and prevents entry of STAT1 into the nucleus [22]. M protein of SARS-CoV interacts with TRF3 and inhibits TBK1/IKKepsilon complex assembly [23]. NSP1 interacts with 40S ribosomal subunit and inactivates the translational activity of the 40S subunits. NSP1-40S ribosome complex can modify the 5' region of capped mRNA template inhibiting the translation of the modified mRNA [24] as well as degradation of mRNA [25]. NSP1 inhibits phosphorylation of STAT1 and represses target genes like ISG15, ISG54, and ISG56 of STAT1 [26]. Inhibition of phosphorylation of IRF3 by ORF3b, ORF6 and N proteins of SARS-CoV and alters the activation of IRF3 [27]. Interaction and inhibition of phosphorylation and nuclear translocation of IRF3 by viral Papain-like protease (PLpro) have been observed [28]. Host protein IRF3 by PLproinhibits de-ubiquitination and changes the stability of IRF3 [29]. These interactions of different viral proteins with IRF3 and its downstream 
STAT1 evade the host immune response. Host-viral protein interactions for SARS-CoV and MERS$\mathrm{CoV}$ are reviewed extensively [19, 30].

Interactions between SARS-CoV-2 proteins and the host proteins are not studied extensively. Very recently interactions of proteins coded by SARS-CoV-2 have been identified. Based on the functions of host interacting partners, it has been proposed that viral proteins coded by SARS-CoV-2 are associated with various functions like DNA replication (NSP1), epigenetic regulation and regulation of gene expression (NSP5, NSP8, NSP13 and E), vesicle trafficking (NSP2, NSP6, NSP7, NSP10, NSP13, NSP15, ORF3a, E, M, ORF8), lipid modification (S), RNA processing and regulation (NSP8, N), ubiquitin ligases (ORF10), signaling (NSP8, NSP13, N, ORF9b), nuclear transport machinery (NSP9, NSP15, ORF6), cytoskeleton function (NSP1, NSP13), mitochondrial functions (NSP4, NSP8, ORF9c) and modification of extracellular matrix (NSP9). In addition, host partners were also associated with various innate immune function, protein synthesis and proteasomal degradation [31] .

\subsection{Knowledge gap}

COVID-19 patients with MetS appear to have severe disease with higher mortality. When infected with SARS-CoV-2, people with MetS have unmasking and/or aggravation of some of its features. Alternatively, pre-existing metabolic conditions might allow infected SARS-CoV-2 to grow aggressively or prevent clearance of the virus due to immune modulation, leading to more severe disease manifestations. Increased viral load and slow clearance of the virus have been reported in severe COVID-19 patients [32, 33]. Severity being associated with MetS, there might be correlation between viral load and slow clearance. SARS-CoV-2 infection triggers stress conditions leading to release of glucocorticoids and catecholamines that increase blood glucose levels [34]. COVID-19 patients with uncontrolled T2D had higher mortality than those with good glycemic control [35]. Two receptors ACE2 and DPP4, necessary for entry of SARS-CoV-2 and MERS-CoV respectively are involved in inflammatory pathways, cardiovascular physiology and glucose homeostasis [36] showing overlap of viral entry, growth and MetS. However, molecular interconnection between COVID-19 severity and MetS remains largely unknown.

\subsection{Hypothesis}

Severity of COVID-19 patients with MetS could be due to functional alterations of host proteins by interacting with viral proteins. 


\section{Material and Methods}

\subsection{SARS viral protein interacting partners of host proteins}

From published data, we collected 609 host proteins that interact with corona virus proteins; specific viral proteins are known for 410 host proteins [19-21, 31, 37]. Detailed sources are shown in the Supplementary Text Table ST2 and Supplementary Table S1.

\subsection{Enrichment analysis}

To find the functional associations of viral interacting proteins, enrichment analysis for the genes was carried out using online facility at Enrichr (https://amp.pharm.mssm.edu/Enrichr/) [17, 18]. Enrichr is an integrative web-based software application for analysis of a gene-set comparing with various geneset libraries. Given an input list of genes, it provides enrichment for different libraries like KEGG pathways, Gene Ontology (GO) terms for biological processes (BPs). DisGeNET, a discovery platform containing publicly available collections of genes and variants associated to human diseases and is also available at the same site.

\subsection{Altered expression of host genes in infected cultured cells}

Altered expression of host genes on infection with virus in different conditions are downloaded from Gene Expression Omnibus (GEO) database, a public functional genomics data repository (https://www.ncbi.nlm.nih.gov/geo/) and catalogued in Enrichr at https://amp.pharm.mssm.edu/Enrichr/ [17, 18]. We collected altered expression of genes for SARS$\mathrm{CoV}$ from this database, the details of which are shown in the Supplementary Table S6

2.4. Functions of the genes whenever necessary were taken from https://www.ncbi.nlm.nih.gov/gene/ and provided by RefSeq.

\subsection{Genes/proteins elevated in organs/tissues}

Datasets for elevated genes and genes expressed in all tissues/organ were collected for 8 organs namely brain, heart, kidney, liver, lung, pancreas, spleen, thyroid and adipose tissue as reported by [38]. Datasets for tissue/organ-specific proteins also collected from data reported by Wilhelm, et al. [39]. Combing these two datasets, unique proteins/genes were determined and designated as elevated 
genes in organ/tissue as well as the housekeeping genes. Expression of genes elevated in different tissues and housekeeping genes are shown in the Supplementary Table S7.

For comparison of different set of genes/proteins, we used online facility at http://bioinformatics.psb.ugent.be/webtools/Venn/

\section{Results}

\subsection{Enriched Biological processes (BPs) with host proteins interacting with SARS virus coded proteins.}

To understand the functional implications of host protein- viral protein interactions, we collected 609 host proteins that interact with proteins coded by SARS-CoV-2 and other corona viruses. Enrichment analysis revealed that 295 BPs enriched significantly (adjusted $p \leq 0.05$ ). The top 10 most significant enriched BPs are shown in Figure 2 and the detail is shown in the Supplementary Table S2.

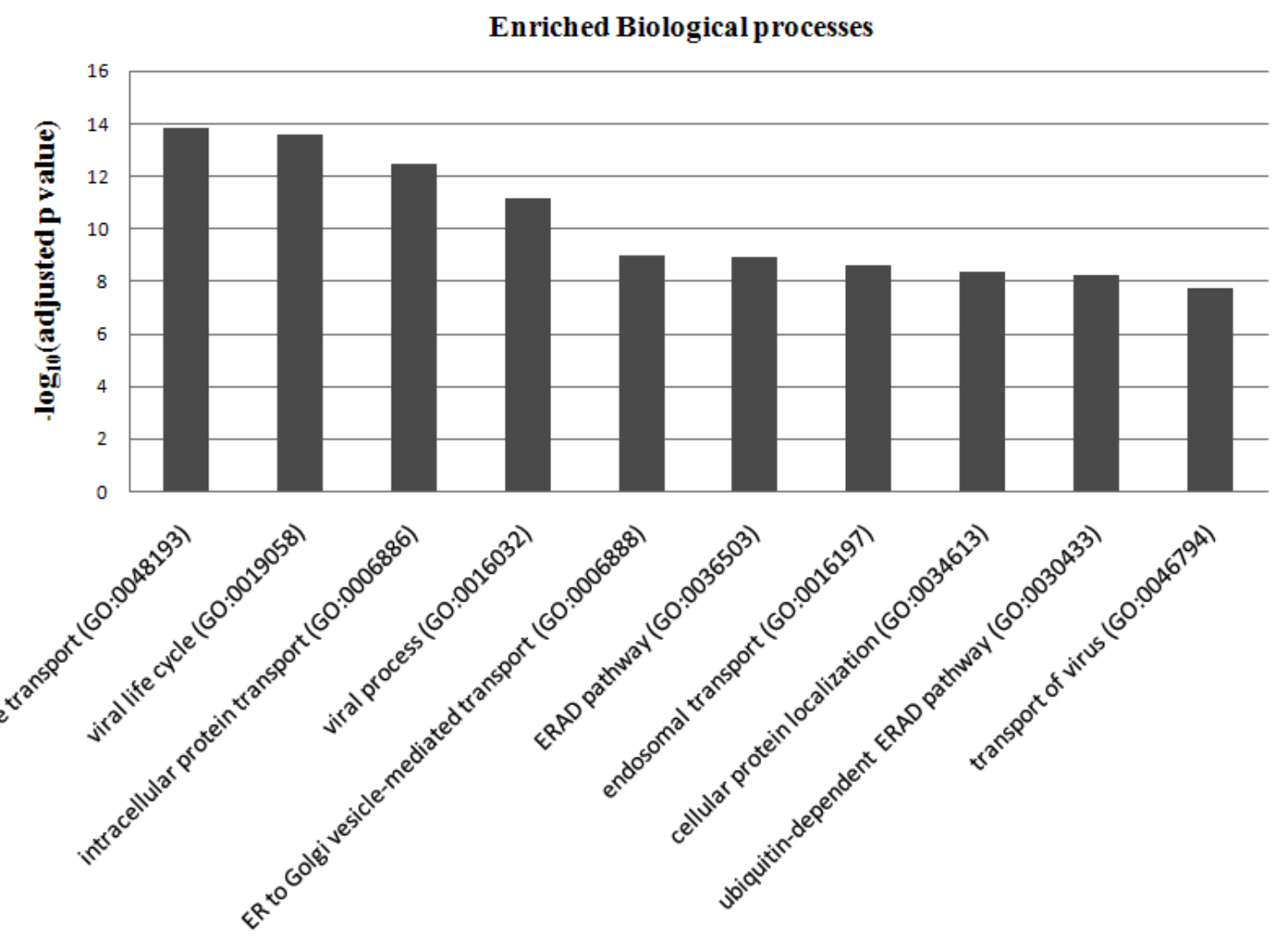

Figure 2: Top 10 significantly enriched biological processes. Golgi vesicle transport (GO:0048193), viral life cycle (GO:0019058), intracellular protein transport (GO:0006886), viral process 
(GO:0016032), ER to Golgi vesicle-mediated transport (GO:0006888), Endoplasmic ReticulumAssociated Degradation (ERAD) pathway (GO:0036503), endosomal transport (GO:0016197), cellular protein localization (GO:0034613), ubiquitin-dependent ERAD pathway (GO:0030433) and transport of virus (GO:0046794) are enriched with 42, 27, 45, 34, 28, 19, 31, 37, 16 and 15 viral protein interacting host protein respectively.

\subsection{Enrichment of KEGG and Wiki Pathways}

Enrichment analysis with 609 interacting proteins revealed that all together 250 KEGG pathways were associated with viral protein interacting host proteins (Supplementary Table S3A); 17 pathways were significantly (adjusted $\mathrm{p} \leq 0.05$ ) enriched with viral protein interacting host proteins (Figure 3). In addition to KEGG pathways, Enrichr also provides enrichment for Wiki Pathways (https://www.wikipathways.org/index.php/WikiPathways). Twenty Wiki pathways like TGF-beta Signaling Pathway (WP366), IL-3 Signaling Pathway (WP286), Hepatitis C and Hepatocellular Carcinoma (WP3646), Viral Acute Myocarditis (WP4298), Sterol Regulatory Element-Binding Proteins (SREBP) signaling (WP1982) and IL-5 Signaling Pathway (WP127) were enriched significantly (adjusted $\mathrm{p} \leq 0.05$ ). Result is shown in the Supplementary Table S3B.

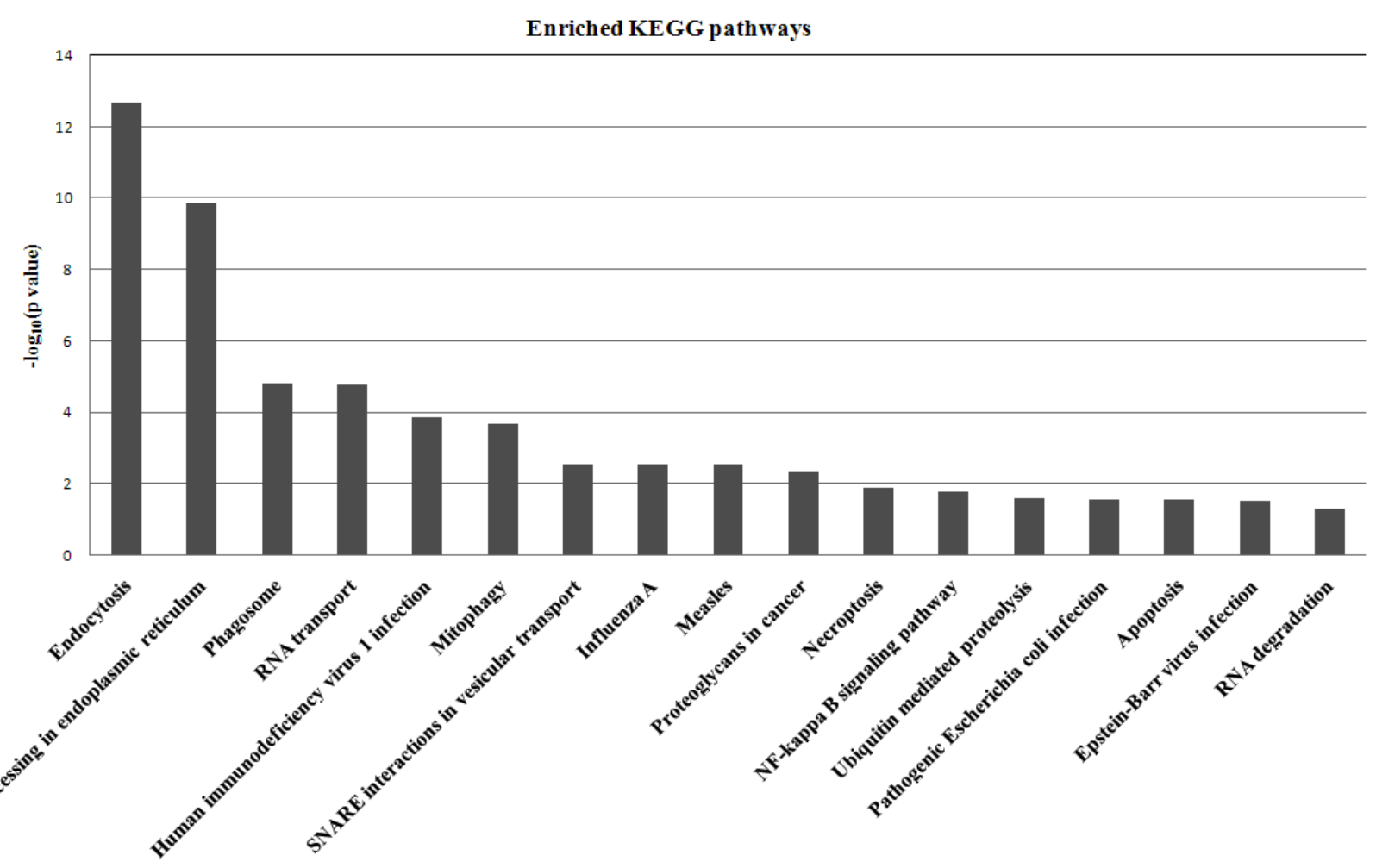


Figure 3: Pathways enriched with SARS-virus interacting proteins.

\subsection{Association of interacting host partners of viral proteins with various metabolic disease/trait catalogued in DisGeNET database}

To identify whether interacting partners of viral proteins are associated with different diseases or disease related conditions/trait, we explored DisGeNET database. This database catalogs gene-disease relations from diverse experimental data like Genome Wide association studies (GWAS) and others [40] as catalogued in Enrichr at https://amp.pharm.mssm.edu/Enrichr/ [17, 18]. Such analysis with viral interacting protein partners revealed that 55 diseases/conditions were significantly (adjusted $\mathrm{p} \leq 0.05)$ associated with viral protein interacting partners; 5308 diseases/conditions were associated with host proteins (Supplementary Table S5A-5C, Figure 4). Many viral interacting host proteins were associated with infection, viruses, metabolic diseases and other diseases including cancers.

$\mathbf{A}$

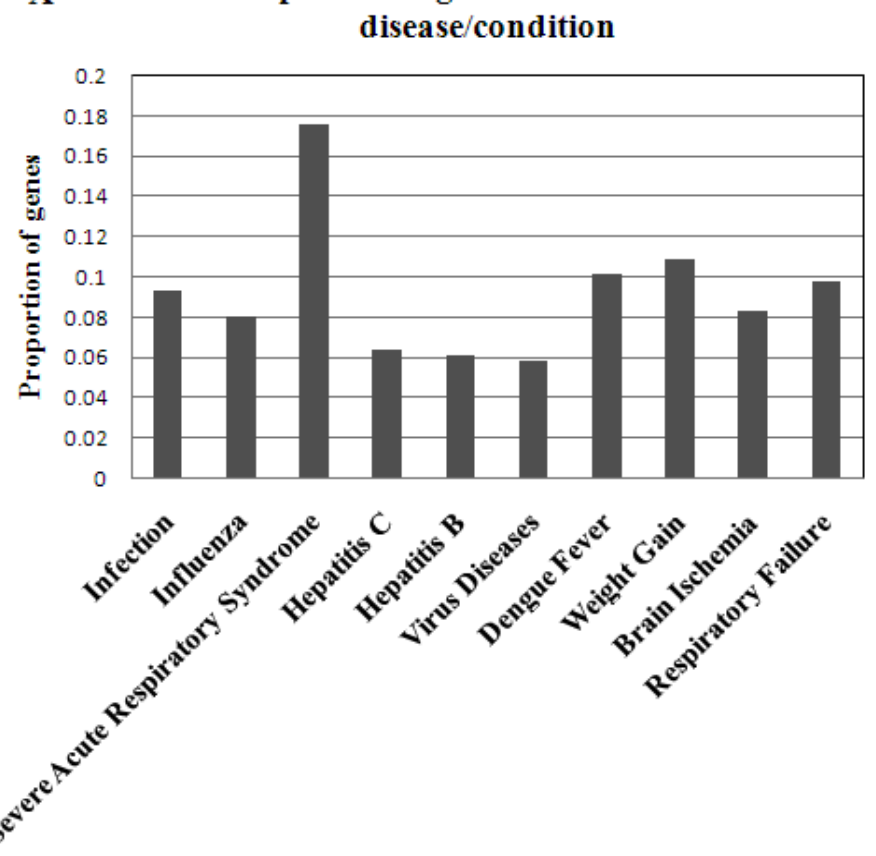

B Proportion of genes associated with
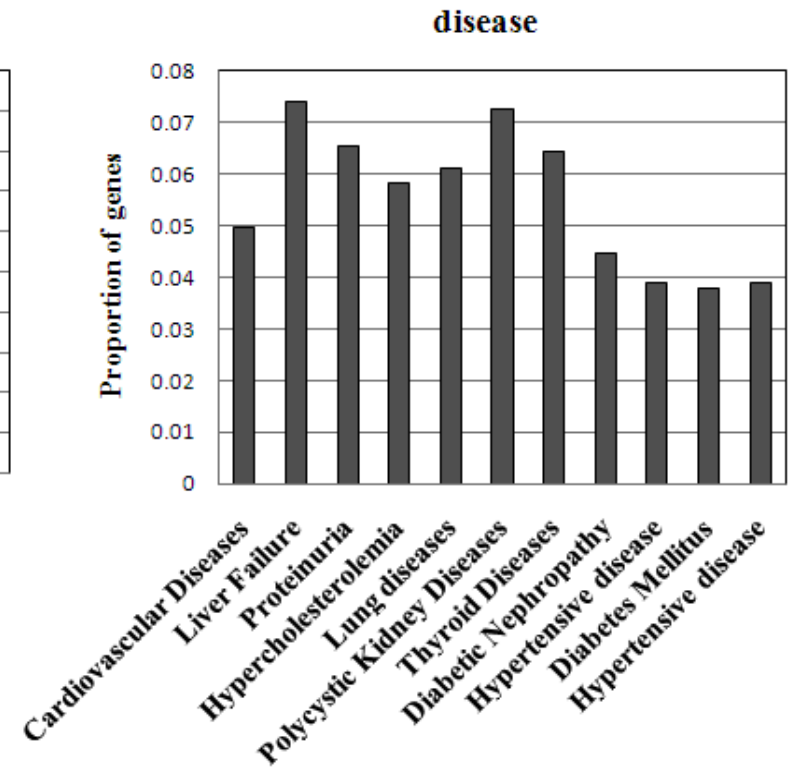

Figure 4: Proportion of genes coding for host protein that interact with proteins coded by SARS-CoV2 and in different categories of diseases/conditions in DisGeNET. For example, total 787 genes are associated in cardiovascular disease. Among 609 protein coded by host genes, 39 proteins are associated with Cardiovascular disease, giving the frequency as 0.05 . Panel A over representation is 
significantly higher (adjusted $\mathrm{p}$ value $\leq 0.05$ ), while the values for the bar diagram in panel $\mathrm{B}$, level of significance is $\leq 0.05$ without correction for multiple testing.

\subsection{Relevant biological processes, pathways and disease terms associated with MetS and enriched with viral protein interacting host proteins}

Various biological processes, pathways and terms in DisGeNET enriched with viral protein interacting host proteins (Supplementary Tables, S2, S3 and S5A) are relevant and known for their involvement in different metabolic diseases. Insulin resistance, insulin signaling pathway, insulin secretion are not only involved in diabetes [41-44] but also in cardiovascular diseases [45] and obesity [46]. The metabolic products of angiotensin I and II play an important role in cardiovascular pathophysiology [47]. Deregulation of lipid metabolism/lipogenesis, fatty acid oxidation (FAO), triglyceride levels, inflammation (adiponectin, TNFa IL6), transcription regulation of lipogenic genes by PPARG in the activation of lipogenic FoxO1 signaling pathway changing glycogenolysis and gluconeogenesis contribute to the development of NAFLD [48] [Figure 5].

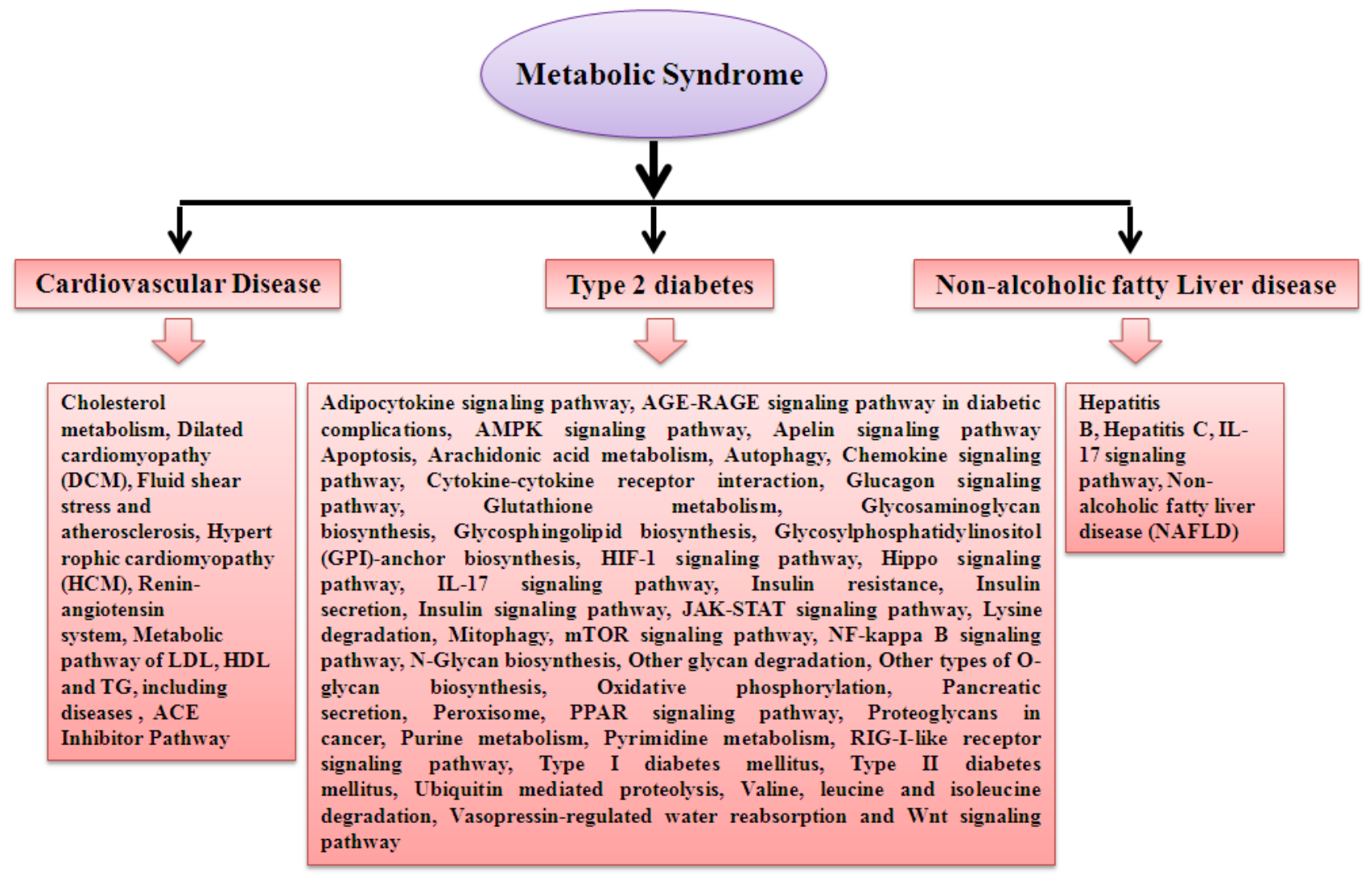


Figure 5 Pathways associated with CVD, diabetes and NAFLD. It is to be noted that even though pathways are shown separately, connected with the diseases, same gene may be associated with different biological processes and pathways.

Biological pathways associated with host genes that interact with different viral proteins were relevant for CVD, diabetes and NAFLD. For example, host protein IKBKB, SLC27A2 and GSK3B interact with coronavirus protein M, NSP2 and (NSP3, N/nucleocapsid protein) respectively and associated with insulin resistance pathway. Similarly, host protein ATP1B1 interacts with $M$ protein and PRKACA interacts with Nsp13/HEL; both the proteins are associated with insulin secretion. GSK3B interacts with viral NSP3 and N/nucleocapsid protein is associated with insulin signaling pathway. Representative result of interactions of viral proteins with host proteins associated with disease relevant pathways is shown in the Figure 6A-6C and Supplementary Text Table ST3. Detailed result is shown in the Supplementary Tables S4A-S4D. 
A

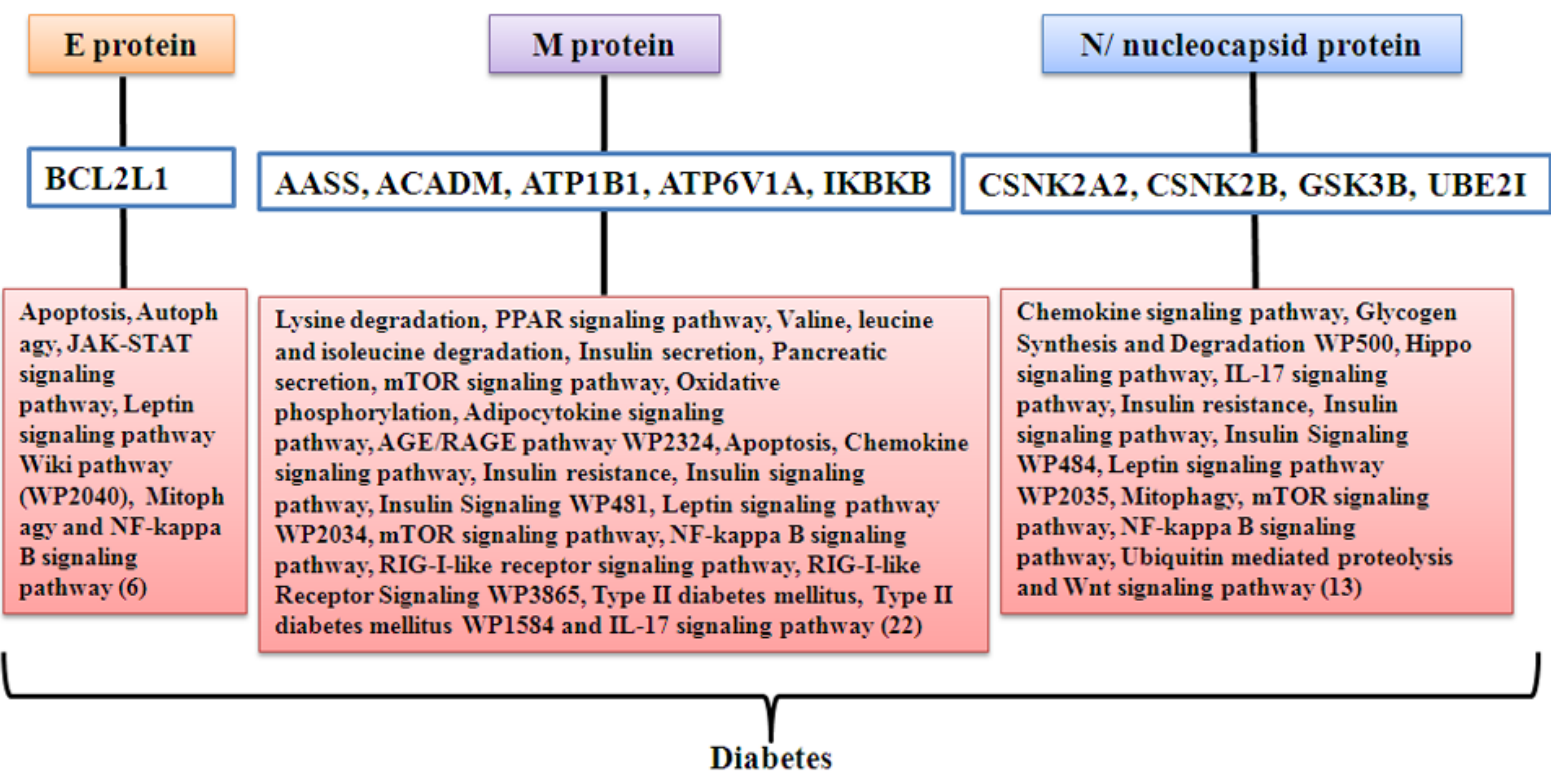

B

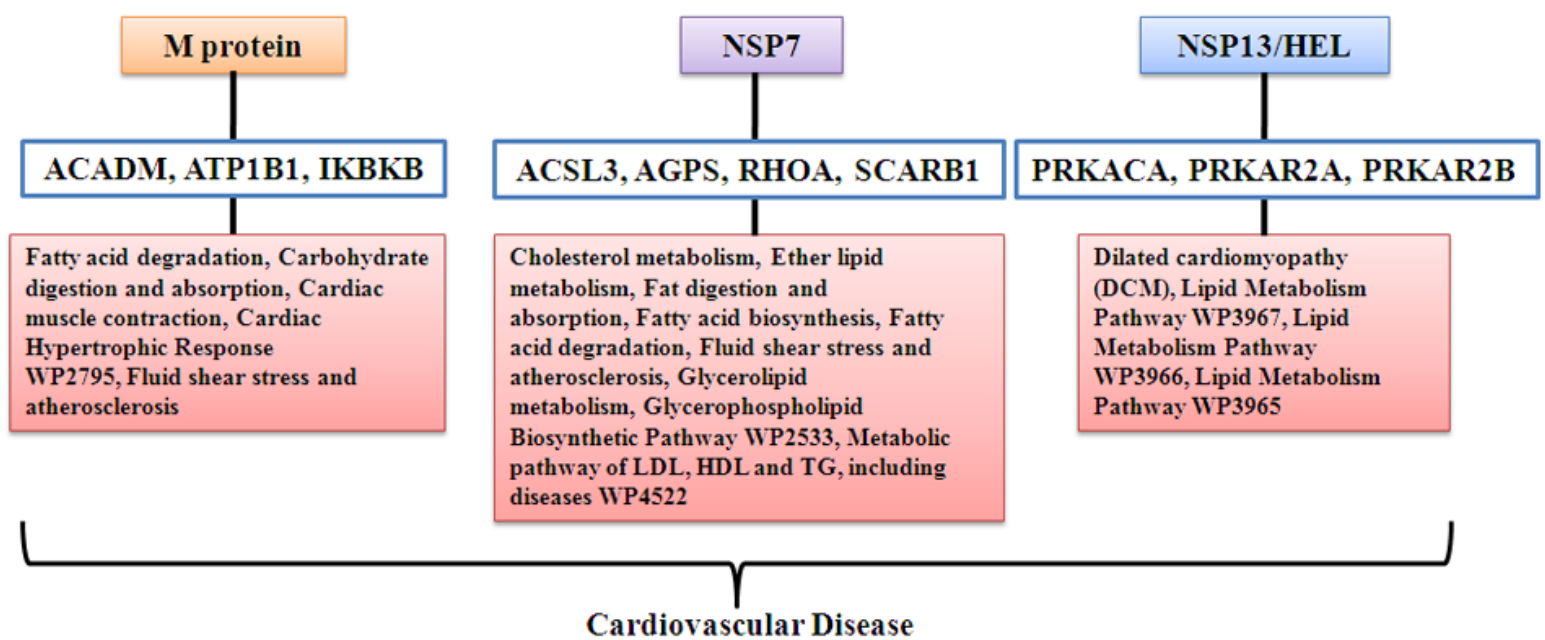

C

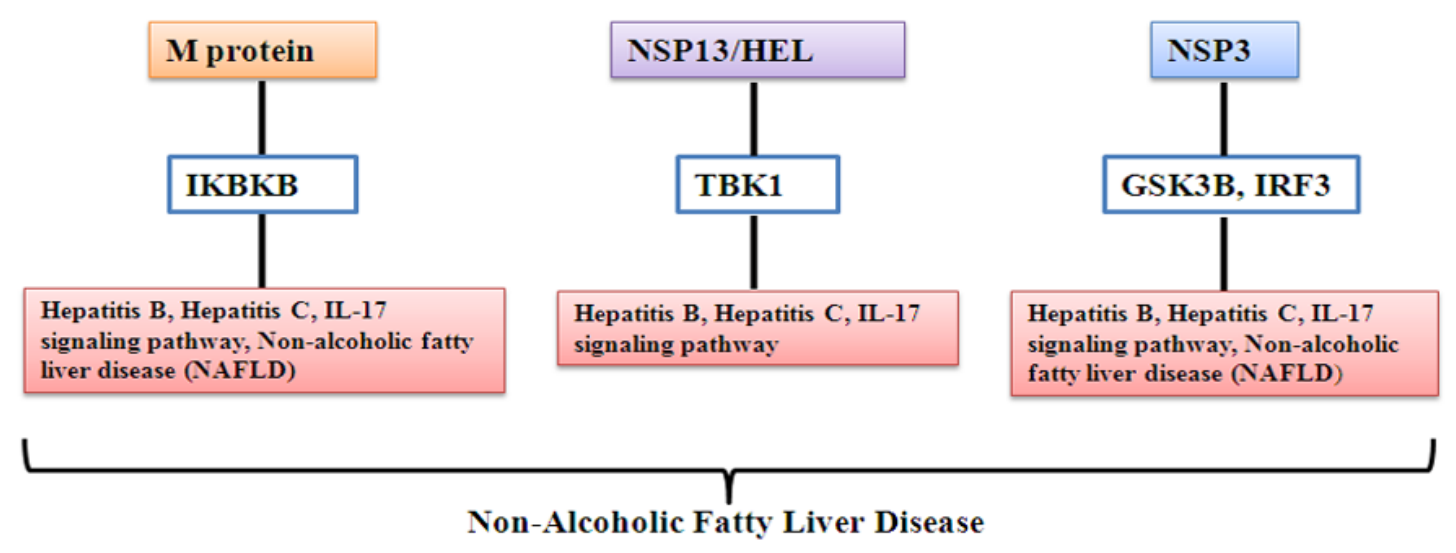

Figure 6 Representative result of viral proteins, their interacting host proteins; host protein associated pathways and involvement in different diseases 
In summary, 27 protein coded by SARS-CoV and SARS-CoV-2 interact with 168 host protein associated with 101 biological processes and pathways related to MetS.

Various host proteins were associated with the diseases or terms linked with diseases in DisGeNET database. Supplementary Figure STF2 shows the representative result of relations of the metabolic diseases, the proteins associated and their interacting partners of viral proteins. Detail result is shown in the supplementary Table S5C.

\subsection{Cytoscape representation of the association of host proteins and viral proteins with diseases in different categories}

A protein categorized in a biological process in Gene Ontology database or in a KEGG pathway that is relevant and known to be involved in a disease is considered to be associated with disease. Similarly, associations of gene/protein with diseases, conditions/traits are catalogued in DisGeNET. Based on our analysis, we categorized biological processes, pathways and disease terms into (i) CVD, (ii) diabetes, (iii) liver disease and (iv) viral infection. To identify the overlap among the various MetS comorbidities mentioned above with host proteins, the free software Cytoscape (version 3.2.1) was used. Their relationships are shown in the Supplementary Tables S8A and S8B and represented using cytoscape in Figures 7 and 8. Result in Figure 7 shows that 16 proteins shown in Green nodes (viz. ACE2, ALB, BCL2, COMT, COX2, IRF3, SMOC1, PPIG, MARK2, IL17RA, NUP210, ITGB1, HMOX1, SCARB1, NUP88 and GPX1) are common for all 4 diseases. In addition, 29 proteins (represented in Pink) are in common for 3 disease nodes, 44 proteins (light yellow squares) overlap 2 disease nodes and the rest (Blue squares) are unique for a given disease. 


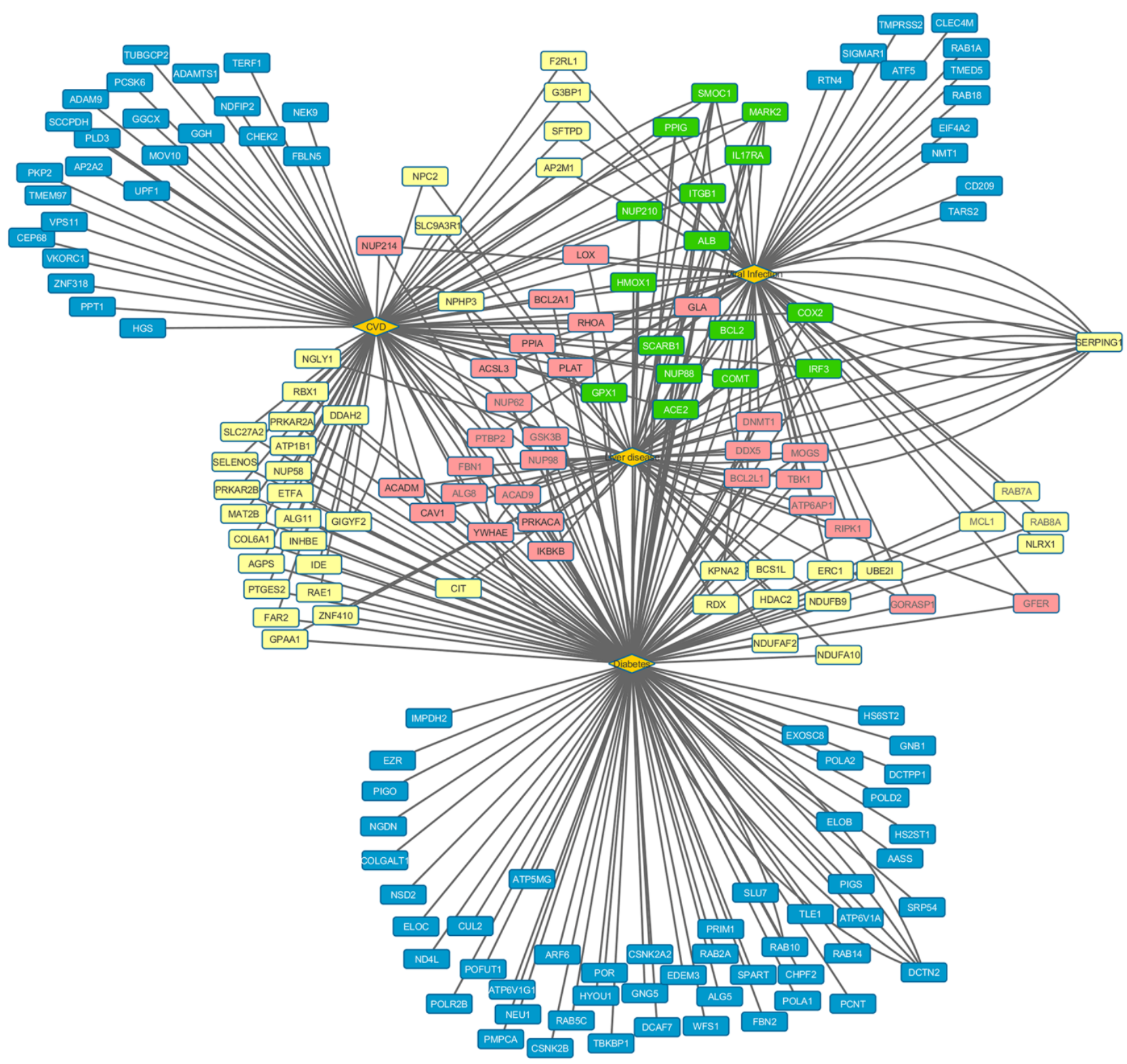

Figure 7: Cytoscape representation of involvement of host protein (rectangle) with CVD, diabetes, Liver disease and viral infection (diamond shape). Green colored host proteins are interacting partners of viral proteins are common for all four diseases, pink colored rectangles represents proteins which are connected with 3 disease, yellow colored rectangles and blue colored rectangles represent viral protein linked to 2 and one disease respectively.

By virtue of interactions of the viral proteins with host proteins, viral proteins are also associated with different diseases. Twenty viral proteins(N protein, M protein, SPIKE/S, NSP1, NSP2, NSP3, NSP4, NSP5, NSP7, NSP8, NSP9, NSP10, NSP12, NSP13, NSP14, ORF3b, ORF6, ORF8, ORF7a, ORF9b, 
OR 9c) were observed to overlap with all four disease modules viz. viral infection, CVD, Diabetes and Liver disease (Figure 8). The viral proteins common for 3 diseases (viz. E proteins, ORF3a, ORF14, and NSP6) are shown in Pink. Light yellow squares represent the viral proteins associated with 2 diseases (NSP15, ORF7b and ORF10). The rest of the viral proteins (Blue squares) are unique for a particular disease. This analysis has strengthened our hypothesis that the frequently occurring comorbidity of MetS could be due to interactions of viral protein with the host proteins.

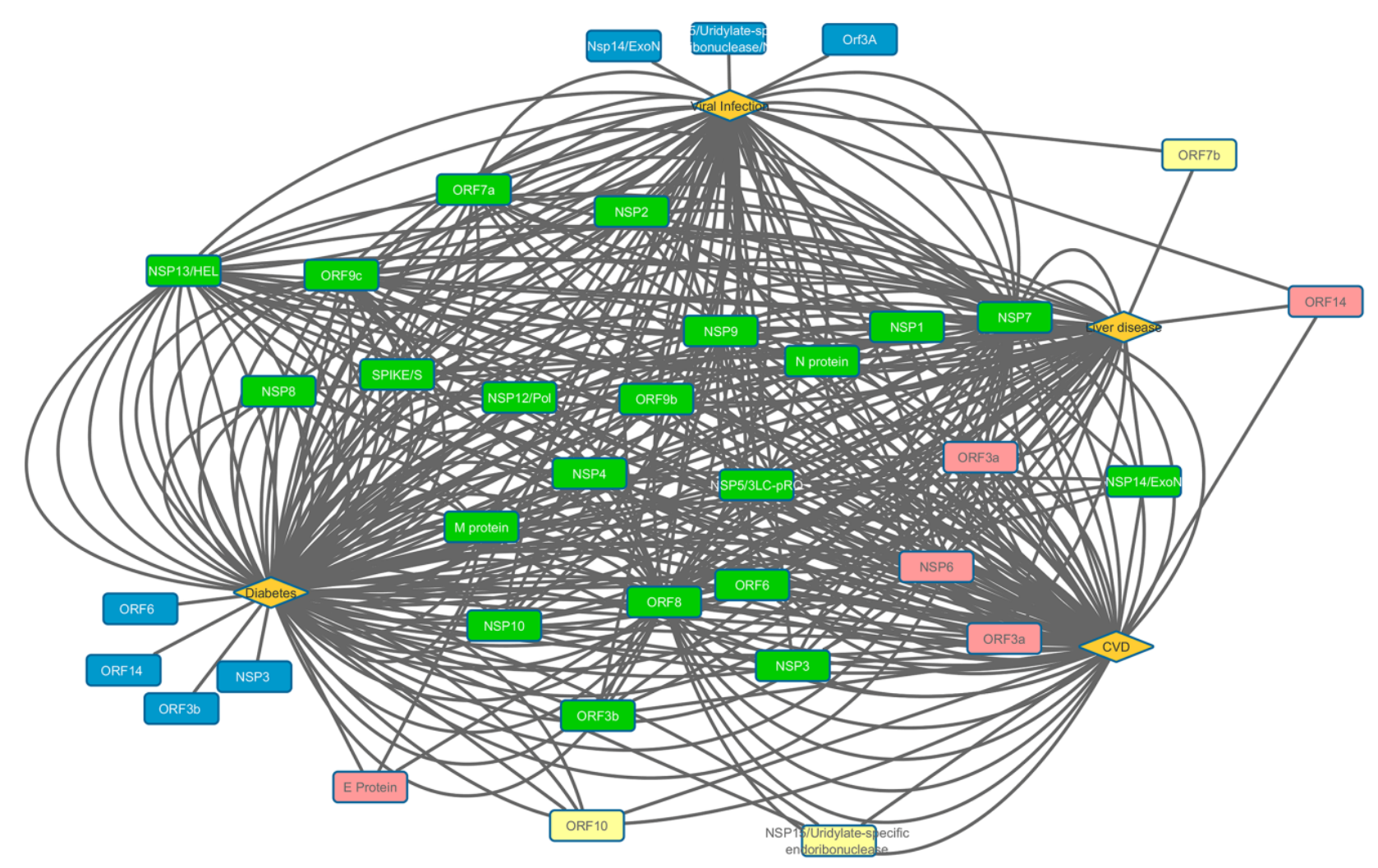

Figure 8: Cytoscape representation of involvement of viral proteins (rectangles) with CVD, diabetes, Liver disease and infectious disease (diamond shape). Green colored viral protein coded by corona virus including MERS-CoV, SARS-CoV and SARS-CoV-2 are common for all four diseases, pink color rectangles represents proteins which are connected with 3 disease, yellow and blue colored rectangles represent viral protein linked to 2 and one disease respectively

\subsection{Organ/tissue enriched expression of host protein interacting partners of SARS-CoV coded protein}


To identify the enriched genes, defined as genes whose mRNA level, determined by RNA sequencing, in a particular tissue is at least five times higher than those in all other tissues, in different organ/tissue like brain, heart, kidney, liver lung, pancreas, spleen and thyroid together with adipose tissue, we used result reported by Uhlén et al. [38]. Gene whose mRNA level is detected in all tissue with fragments per kilobase of exon model per million reads mapped greater than 1 is considered to be housekeeping genes [38]. We used these organ/tissue enriched and compared with viral protein interacting partners, to identify whether viral protein interacting partner of the host gene enhanced in any particular tissue/organ. The result is shown in the Supplementary Text Table ST4. Majority of genes coded for viral interacting proteins are housekeeping gene and expressed in all tissues. Only a small portion of the genes are enriched with different tissues like heart, lung, pancreas, adipose tissue, kidney, and liver. It requires further investigations whether this small number of organ/tissue enriched proteins contributes to the organ/tissue specific pathogenic effects of COVID-19.

\subsection{Altered expression of host genes after infection with SARS-CoV}

Combined proteome and transcriptome datasets from infection of SARS-CoV in human lung epithelial cells in culture are utilized to predict regulatory genes involved in the host response. After infection expression of genes were determined at different time points [49]. Increased and decreased expressions of the genes are taken from Enrchr. Summary of the result is shown in the Table and the detail is shown in the supplementary Text Table T2. Overall about $5 \%$ of the increased expression and similar proportion of decreased expression of host genes in SARS-CoV infected cells also interact with viral proteins. Out of 609 viral protein interacting host proteins, expression of about $28 \%$ genes coded for the viral protein interacting partners increased and expression of $36 \%$ genes decreased; overall expression of $64 \%$ of genes altered (Table 3). Mechanism of deregulation of host genes by viral infection remains largely unknown. Deregulation and physical interaction of deregulated host genes, support the general notion that deregulated genes products interact with each other and carry out biological functions. 
Table 3: Deregulated genes after infection with SARS-CoV and interactions of viral proteins with the host proteins

\begin{tabular}{|l|l|l|l|l|}
\hline \multirow{2}{*}{$\begin{array}{l}\text { Time after } \\
\text { infection }\end{array}$} & \multicolumn{2}{|l|}{ Total number of genes deregulated } & \multicolumn{2}{l|}{ No. Viral protein interacting protein } \\
\cline { 2 - 5 } & Increased & Decreased & Increased (\%) & Decreased (\%) \\
\hline $0 \mathrm{~h}$ & 581 & 694 & $28(5.8)$ & $41(5.9)$ \\
\hline $12 \mathrm{~h}$ & 637 & 609 & $27(4.2)$ & $41(6.7)$ \\
\hline $24 \mathrm{~h}$ & 763 & 898 & $32(4.2)$ & $75(8.3)$ \\
\hline $36 \mathrm{~h}$ & 197 & 218 & $18(9.1)$ & $15(6.9)$ \\
\hline $48 \mathrm{~h}$ & 740 & 822 & $28(3.8)$ & $39(4.7)$ \\
\hline $60 \mathrm{~h}$ & 616 & 662 & $25(4.1)$ & $37(5.6)$ \\
\hline $72 \mathrm{~h}$ & 579 & 581 & $31(5.4)$ & $43(7.4)$ \\
\hline $84 \mathrm{~h}$ & 587 & 585 & $25(4.2)$ & $30(5.1)$ \\
\hline $96 \mathrm{~h}$ & 622 & 587 & $38(6.1)$ & $34(5.8)$ \\
\hline Total & 3465 & 3598 & $170(4.9)$ & $220(6.1)$ \\
\hline
\end{tabular}

\section{Discussion}

To find out the molecular basis of severity of COVI-19 with MetS, we collected data from various sources for host protein interacting partners of proteins coded by SARS-CoV, SARS-CoV-2 and other corona viruses and analyzed using various online tools. Such analysis revealed (i) possible role of host proteins in life cycle of the virus and (ii) viral protein interacting host proteins are enriched with various biological processes and pathways relevant for CVD, diabetes and NAFLD. Besides many host genes coding for viral interacting partners are associated with MetS or related traits in DisGeNET. This result shows that COVID-19 and MetS might be associated through the proteins involved in MetS and are also targets of viral proteins.

\subsection{Mechanism (s) of severity of COVID-19 in patients with MetS}

Severity and mortality of COVID-19 is associated with MetS. Increased viral load and decreased clearance of viruses are major contributors to COVID-19 related morbidity and mortality [32, 33]. Viral load for COVID-19 patient comorbid with MetS is not known. Possible increased viral load and slow clearance in COVID-19 patients with MetS, if observed, might explain the higher death rate and poor outcomes in them. Infection with SARS-CoV-2 in subjects with MetS, a state of chronic low 
grade inflammation, may trigger the release of catecholamines and glucocorticoids that further increase blood glucose levels and increase glucose variability [34]. Hyperglycemia and insulin resistance are known to promote enhanced synthesis of glycation end products (AGEs), proinflammatory cytokines and adhesion molecules that may interact with viral proteins and worsen outcome in COVID-19 [50, 51]. Thus, infection enhances the metabolic conditions which in turn increase pathogenicity of the virus by modulating AGEs and pro-inflammatory cytokines.

In MetS, immune system is compromised due to defects in inflammation. This condition might delay in clearing viruses and recovery. MERS-CoV infection to diabetic mice results in immune deregulation and enhances disease severity of the infection [52]. Virus RNA was detected in lungs of $100 \%$ of the COVID-19 patients, while in about $40 \%$ of the patients viral RNA was detected in heart, liver and kidney, possibly reached through blood [53]. Increased levels of troponin levels, a sensitive marker for acute cardiac injury, is commonly observed in severe cases of COVID-19 and is strongly associated with mortality [54]. Acute coronary syndrome and myocardial infarction were noted to occur after SARS [55]. So far no convincing reports are available to show that SARS-CoV-2 infects pancreatic cells [56] or causes acute pancreatitis by the infection [57]. However, effects of direct infection of SARS-CoV in pancreas inducing diabetes in non-diabetic persons are known [58].

\subsection{Common genes in COVID-19 and MetS}

Sixteen genes are related to infectious diseases, CVD, diabetes and liver diseases (Figure 7). Relevance of ACE2, ALB, ITGB1, IRF3, MARK2, IL17RA, HMOX1, SCARB1, GPX1 and BCL2 genes for infections and MetS are known. ACE2 has been implicated in obesity, hypertension, diabetes, myocardial infarction, heart failure and inflammatory lung disease [59-61]. ACE2 has been proposed to be the receptor for entry into the host cells by interacting with the spike protein of SARSCoV-2 [62] and SARS-CoV, possibly through renin-angiotensin system as shown for SARS-CoV [63]. Serum albumin levels are decreased in infection, liver dysfunction, obesity, diabetes and CVD due to its ability to act as antioxidant [64, 65], modify inflammation, cytokine production [66] and maintain capillary membrane stability and fluid balance across the capillary [67]. ITGB1, a member of integrin beta chain family, promotes entry of different viruses [68], although its role in SARS-CoV-2 remains unknown. ITGB activation enhances secretion of insulin and inhibited by excess glucose [69]. Interferon regulatory factor 3 (IRF3) modulates innate immune response to viral and bacterial infections [70], inflammation [71], glucose and lipid homeostasis [72, 73] by interacting with viral proteins and regulating interferon genes. IL17RA by altering downstream cytokines and chemokine 
involves in infectious disease and reviewed [74], diabetes [75] and heart failure [76]. Evidences that the IL-17 signaling pathway contributes to the pathogenesis of liver diseases by inhibiting fatty acid $\beta$ oxidation and altering cell death [77], ERK1/2/p65 signaling pathway [78], obesity, inflammation and reviewed [79] are available. Modified IL-17 signaling pathway has been observed in chronic viral hepatitis [80]. SCARB1, a receptor for high density lipoproteins, facilitates uptake of cholesteryl esters from HDL to the liver. It has been implicated in entry of HCV [81]. Level of serum HDL-Cholesterol is risk factor for CVD [82, 83], hypertension, hyperlipidemia, insulin resistance and obesity [84]. Microtubule Affinity Regulating Kinase 2 (MARK2) involves in motility and uncoatng HIV-1 by locally phosphorylating the viral cores [85]. It is one of the kinases that phosphorylates AMPK and involves in epithelial mesenchymal transition [86], metabolic rate, adiposity, and insulin hypersensitivity [87]. HMOX1 possess cytoprotective, anti-inflammatory and antioxidant properties and modulates HBV infection [88], fatty liver, NASH, NAFL, insulin resistance, hypertension, vascular dysfunction, diabetes, atheroscerosis and others [89]. Glutathione peroxidase 1 (GPX-1) is an enzyme having antioxidant properties and involves in coxsackie virus induced myocarditis [90], influenza A virus-induced lung inflammation [91], HCV induced liver fibrosis and heptocellular carcinoma [92] and HBB replication [93]. Increased oxidative stress in MetS and a decreased antioxidative defense were correlated with triglycerides, high-density lipoprotein cholesterol, waist circumference, blood pressure components of MetS [94]. There are reports to show that mortality due to CVD is associated with low serum level selenium. Low selenium levels also correlate with risk of myocardial infarction. Contradictory result of no effect or increased risk of ischemic heart disease ((reviewed in Bellinger, et al. [95]) has also been reported. Even though various proteins identified here have common role in viral infections and MetS, whether these are relevant for SARS-CoV-2 infection remains unknown and further studies are necessary. Taking together, our analysis provides molecular clues for over representation of MetS in COVID-19 patients, especially for severe disease and/or or non-survivors.

Altered gene expression from peripheral blood in MetS shows a general systemic inflammation condition mediated by the innate immune system. NF- $\mathrm{kB}$ activation, activation of $\mathrm{T}$ cells, macrophages and increased expression of CXCL14, IL4R, GPX1 and many others are observed in MetS, diabetes and coronary heart disease [96]. In pre-existing MetS condition, when infection with SARS-CoV-2 occurs, viral coded proteins interact with host proteins that have already been compromised or activated. Such interactions might result in functional interactions synergistically 
between the infection and pre-existing conditions. This condition might delay in clearing viruses and recovery as observed for MERS-CoV infection to diabetic mice [52]. It remains unknown whether infection with SARS-CoV-2 in individual without any comorbidity induces acute injury to heart, liver or pancreas either due to direct infection in these organ or by systemic changes in inflammation and immune modulation. Acute cardiac injury is strongly associated with mortality and severity [54]. SARS-CoV coded structural protein 7 a can cause direct liver injury by inducing apoptosis in vitro studies [97]. Liver injury has also been observed in COVID-19 patients [98, 99].

\section{Conclusion}

SARS-CoV-2 coded protein interacting host proteins are associated with CVD, diabetes, or NAFLD relevant biological processes, pathways and diseases/traits. Interaction of the viral proteins alters the functions of their host partners for the survival of viruses. In absence of infection, functional changes in these proteins, possibly through expression, result in MetS. SARS-CoV-2 infection in patients with metabolic diseases like CVD, diabetes or NAFLD, might synergistically modify systemic functions of these common genes resulting in severity and mortality of COVID-19 patients. Direct infection of SARS-CoV-2 in heart and liver might also result in localized as well as systemic functional changes of the proteins giving rise severity of the disease. Induction of acute injury to liver, heart or pancreas either due to direct infection of the virus or systemic damage by SARS-CoV-2 infection of normal individuals and their long term effects, if any, remain unknown. Such analysis not only provided molecular basis of comorbidity but also knowledge about the role of host proteins in viral replication and growth and possible drug targets for intervention. Further studies are necessary to correlate with altered gene expression in diabetes, CVD, hypertension, obese and other metabolic conditions with altered gene expression in viral infected tissues. It also remains unknown whether people with MetS are susceptible to SARS-CoV-2 infection or infection with SARS-CoV-2 made people prone to MetS.

\section{Funding}

This research did not receive any specific grant from funding agencies in the public, commercial, or not-for-profit sectors.

\section{Disclosure of potential conflicts of interest}

The authors declare no conflict of interest. 


\section{References}

[1] F. Zhou, T. Yu, R. Du, et al., Clinical course and risk factors for mortality of adult inpatients with COVID-19 in Wuhan, China: a retrospective cohort study, The lancet, 395 (2020) 1054-1062, doi: https://doi.org/10.1016/S01406736(20)30566-3.

[2] M. Yuan, W. Yin, Z. Tao, et al., Association of radiologic findings with mortality of patients infected with 2019 novel coronavirus in Wuhan, China, Plos one, 15 (2020) e0230548, doi: https://doi.org/10.1371/journal.pone.0230548.

[3] J. Yang, Y. Zheng, X. Gou, et al., Prevalence of comorbidities and its effects in patients infected with SARS-CoV-2: a systematic review and meta-analysis, International journal of infectious diseases, 94 (2020) 91-95, doi: 10.1016/j.ijid.2020.03.017.

[4] T. Chen, Z. Dai, P. Mo, et al., Clinical characteristics and outcomes of older patients with coronavirus disease 2019 (COVID-19) in Wuhan, China (2019): a single-centered, retrospective study, The journals of gerontology. Series A, Biological sciences and medical sciences (2020), doi: 10.1093/gerona/glaa089.

[5] C. Huang, Y. Wang, X. Li, et al., Clinical features of patients infected with 2019 novel coronavirus in Wuhan, China, The lancet, 395 (2020) 497-506, doi: https://doi.org/10.1016/S0140-6736(20)30183-5.

[6] S. Garg, L. Kim, M. Whitaker, et al., Hospitalization Rates and Characteristics of Patients Hospitalized with Laboratory-Confirmed Coronavirus Disease 2019 - COVID-NET, 14 States, March 1-30, 2020, MMWR Morb Mortal Wkly Rep, 69 (2020) 458-464, doi: 10.15585/mmwr.mm6915e3.

[7] G. Onder, G. Rezza, S. Brusaferro, Case-Fatality Rate and Characteristics of Patients Dying in Relation to COVID-19 in Italy, Jama, 323 (2020) 1775-1776, doi: 10.1001/jama.2020.4683.

[8] D. Wang, B. Hu, C. Hu, et al., Clinical Characteristics of 138 Hospitalized Patients With 2019 Novel CoronavirusInfected Pneumonia in Wuhan, China, JAMA, 323 (2020) 1061-1069, doi: 10.1001/jama.2020.1585.

[9] R.E. Jordan, P. Adab, K.K. Cheng, Covid-19: risk factors for severe disease and death, BMJ, 368 (2020) m1198, doi: 10.1136/bmj.m1198.

[10] C.C.f.D.C. Epidemiology Working Group for Ncip Epidemic Response, Prevention, [The epidemiological characteristics of an outbreak of 2019 novel coronavirus diseases (COVID-19) in China], Zhonghua liu xing bing xue za zhi = Zhonghua liuxingbingxue zazhi, 41 (2020) 145-151, doi: 10.3760/cma.j.issn.0254-6450.2020.02.003.

[11] J. Lighter, M. Phillips, S. Hochman, et al., Obesity in Patients Younger Than 60 Years Is a Risk Factor for COVID-19 Hospital Admission, Clinical Infectious Diseases, (2020), doi: 10.1093/cid/ciaa415.

[12] Y. Dong, H. Chen, J. Gao, et al., Molecular machinery and interplay of apoptosis and autophagy in coronary heart disease, Journal of molecular and cellular cardiology, 136 (2019) 27-41, doi: 10.1016/j.yjmcc.2019.09.001.

[13] S. O'Neill, L. O'Driscoll, Metabolic syndrome: a closer look at the growing epidemic and its associated pathologies, Obesity reviews : an official journal of the International Association for the Study of Obesity, 16 (2015) 1-12, doi: 10.1111/obr.12229.

[14] C.A. Aguilar-Salinas, T. Viveros-Ruiz, Recent advances in managing/understanding the metabolic syndrome, F1000Research, 8 (2019), doi: 10.12688/f1000research.17122.1.

[15] S.A. Heialy, M. Hachim, A. Senok, et al., Regulation of angiotensin converting enzyme 2 (ACE2) in obesity: implications for COVID-19, bioRxiv, (2020) 2020.2004.2017.046938, doi: 10.1101/2020.04.17.046938. 
[16] D. Blanco-Melo, B.E. Nilsson-Payant, W.-C. Liu, et al., Imbalanced Host Response to SARS-CoV-2 Drives Development of COVID-19, Cell, 181 (2020) 1036-1045.e1039, doi: https://doi.org/10.1016/j.cell.2020.04.026.

[17] E.Y. Chen, C.M. Tan, Y. Kou, et al., Enrichr: interactive and collaborative HTML5 gene list enrichment analysis tool, BMC Bioinformatics, 14 (2013) 128, doi: 10.1186/1471-2105-14-128.

[18] M.V. Kuleshov, M.R. Jones, A.D. Rouillard, et al., Enrichr: a comprehensive gene set enrichment analysis web server 2016 update, Nucleic Acids Res, 44 (2016) W90-97, doi: 10.1093/nar/gkw377.

[19] T.S. Fung, D.X. Liu, Human Coronavirus: Host-Pathogen Interaction, Annu Rev Microbiol, 73 (2019) 529-557, doi: 10.1146/annurev-micro-020518-115759.

[20] P. V'kovski, M. Gerber, J. Kelly, et al., Determination of host proteins composing the microenvironment of coronavirus replicase complexes by proximity-labeling, Elife, 8 (2019) e42037, doi: 10.7554/eLife.42037.

[21] S. Pfefferle, J. Schöpf, M. Kögl, et al., The SARS-coronavirus-host interactome: identification of cyclophilins as target for pan-coronavirus inhibitors, PLoS pathogens, 7 (2011), doi: 10.1371/journal.ppat.1002331.

[22] M. Frieman, B. Yount, M. Heise, et al., Severe acute respiratory syndrome coronavirus ORF6 antagonizes STAT1 function by sequestering nuclear import factors on the rough endoplasmic reticulum/Golgi membrane, Journal of virology, 81 (2007) 9812-9824, doi: 10.1128/jvi.01012-07.

[23] K.L. Siu, K.H. Kok, M.H. Ng, et al., Severe acute respiratory syndrome coronavirus M protein inhibits type I interferon production by impeding the formation of TRAF3.TANK.TBK1/IKKepsilon complex, The Journal of biological chemistry, 284 (2009) 16202-16209, doi: 10.1074/jbc.M109.008227.

[24] W. Kamitani, C. Huang, K. Narayanan, et al., A two-pronged strategy to suppress host protein synthesis by SARS coronavirus Nsp1 protein, Nature structural \& molecular biology, 16 (2009) 1134-1140, doi: 10.1038/nsmb.1680.

[25] W. Kamitani, K. Narayanan, C. Huang, et al., Severe acute respiratory syndrome coronavirus nsp1 protein suppresses host gene expression by promoting host mRNA degradation, Proceedings of the National Academy of Sciences, 103 (2006) 12885-12890, doi: 10.1073/pnas.0603144103.

[26] M.G. Wathelet, M. Orr, M.B. Frieman, et al., Severe acute respiratory syndrome coronavirus evades antiviral signaling: role of nsp1 and rational design of an attenuated strain, Journal of virology, 81 (2007) 11620-11633, doi: 10.1128/jvi.00702-07.

[27] S.A. Kopecky-Bromberg, L. Martínez-Sobrido, M. Frieman, et al., Severe acute respiratory syndrome coronavirus open reading frame (ORF) 3b, ORF 6, and nucleocapsid proteins function as interferon antagonists, Journal of virology 81 (2007) 548-557, doi: 10.1128/jvi.01782-06.

[28] S.G. Devaraj, N. Wang, Z. Chen, et al., Regulation of IRF-3-dependent innate immunity by the papain-like protease domain of the severe acute respiratory syndrome coronavirus, The Journal of biological chemistry, 282 (2007) 3220832221, doi: 10.1074/jbc.M704870200.

[29] K. Matthews, A. Schäfer, A. Pham, et al., The SARS coronavirus papain like protease can inhibit IRF3 at a post activation step that requires deubiquitination activity, Virology Journal, 11 (2014) 209, doi: 10.1186/s12985-014-0209-9.

[30] Y. Chen, Q. Liu, D. Guo, Emerging coronaviruses: genome structure, replication, and pathogenesis, Journal of medical virology, 92 (2020) 418-423, doi: https://doi.org/10.1002/jmv.25681. 
[31] D.E. Gordon, G.M. Jang, M. Bouhaddou, et al., A SARS-CoV-2 protein interaction map reveals targets for drug repurposing, Nature, (2020), doi: 10.1038/s41586-020-2286-9.

[32] Y.-Y. Zheng, Y.-T. Ma, J.-Y. Zhang, et al., COVID-19 and the cardiovascular system, Nature Reviews Cardiology, 17 (2020) 259-260, doi: https://doi.org/10.1038/s41569-020-0360-5.

[33] Y. Liu, L.M. Yan, L. Wan, et al., Viral dynamics in mild and severe cases of COVID-19, Lancet Infect Dis, 20 (2020) 656-657, doi: 10.1016/s1473-3099(20)30232-2.

[34] A. Wang, W. Zhao, Z. Xu, et al., Timely blood glucose management for the outbreak of 2019 novel coronavirus disease (COVID-19) is urgently needed, Diabetes research and clinical practice, 162 (2020) 108118, doi: 10.1016/j.diabres.2020.108118.

[35] L. Zhu, Z.G. She, X. Cheng, et al., Association of Blood Glucose Control and Outcomes in Patients with COVID-19 and Pre-existing Type 2 Diabetes, Cell metabolism, 31 (2020) 1068-1077, doi: 10.1016/j.cmet.2020.04.021.

[36] D.J. Drucker, Coronavirus Infections and Type 2 Diabetes-Shared Pathways with Therapeutic Implications, Endocrine reviews, 41 (2020), doi: 10.1210/endrev/bnaa011.

[37] Y. Zhou, Y. Hou, J. Shen, et al., Network-based drug repurposing for novel coronavirus 2019-nCoV/SARS-CoV-2, Cell discovery, 6 (2020) 1-18, doi: https://doi.org/10.1038/s41421-020-0153-3.

[38] M. Uhlén, L. Fagerberg, B.M. Hallström, et al., Proteomics. Tissue-based map of the human proteome, Science, 347 (2015) 1260419, doi: 10.1126/science.1260419.

[39] M. Wilhelm, J. Schlegl, H. Hahne, et al., Mass-spectrometry-based draft of the human proteome, Nature, 509 (2014) 582-587, doi: 10.1038/nature13319.

[40] J. Piñero, J.M. Ramírez-Anguita, J. Saüch-Pitarch, et al., The DisGeNET knowledge platform for disease genomics: 2019 update, Nucleic Acids Res, 48 (2020) D845-d855, doi: 10.1093/nar/gkz1021.

[41] R. Taylor, Insulin Resistance and Type 2 Diabetes, Diabetes, 61 (2012) 778-779, doi: 10.2337/db12-0073.

[42] J. Boucher, A. Kleinridders, C.R. Kahn, Insulin receptor signaling in normal and insulin-resistant states, Cold Spring Harb Perspect Biol, 6 (2014) a009191, doi: 10.1101/cshperspect.a009191.

[43] B.I. Posner, Insulin Signalling: The Inside Story, Canadian journal of diabetes, 41 (2017) 108-113, doi: 10.1016/j.jcjd.2016.07.002.

[44] P.J. Guillausseau, T. Meas, M. Virally, et al., Abnormalities in insulin secretion in type 2 diabetes mellitus, Diabetes \& Metabolism, 34 (2008) S43-S48, doi: https://doi.org/10.1016/S1262-3636(08)73394-9.

[45] Z. Bloomgarden, Y. Handelsman, World congress on insulin resistance, diabetes and cardiovascular disease, Journal of diabetes, 10 (2018) 776-777, doi: 10.1111/1753-0407.12787.

[46] E.J. Gallagher, D. Leroith, E. Karnieli, Insulin resistance in obesity as the underlying cause for the metabolic syndrome, The Mount Sinai journal of medicine, 77 (2010) 511-523, doi: 10.1002/msj.20212.

[47] M. Paz Ocaranza, J.A. Riquelme, L. García, et al., Counter-regulatory renin-angiotensin system in cardiovascular disease, Nature Reviews Cardiology, 17 (2020) 116-129, doi: 10.1038/s41569-019-0244-8.

[48] A. Berlanga, E. Guiu-Jurado, J.A. Porras, et al., Molecular pathways in non-alcoholic fatty liver disease, Clinical and experimental gastroenterology, 7 (2014) 221-239, doi: 10.2147/ceg.s62831. 
[49] H.D. Mitchell, A.J. Eisfeld, A.C. Sims, et al., A network integration approach to predict conserved regulators related to pathogenicity of influenza and SARS-CoV respiratory viruses, PLoS One, 8 (2013), doi: 10.1371/journal.pone.0069374. [50] S. Knapp, Diabetes and infection: is there a link?--A mini-review, Gerontology, 59 (2013) 99-104, doi: 10.1159/000345107.

[51] J.R. Petrie, T.J. Guzik, R.M. Touyz, Diabetes, Hypertension, and Cardiovascular Disease: Clinical Insights and Vascular Mechanisms, The Canadian journal of cardiology, 34 (2018) 575-584, doi: 10.1016/j.cjca.2017.12.005.

[52] K.A. Kulcsar, C.M. Coleman, S.E. Beck, et al., Comorbid diabetes results in immune dysregulation and enhanced disease severity following MERS-CoV infection, JCI Insight, 4 (2019) e131774, doi: 10.1172/jci.insight.131774.

[53] D. Wichmann, J.-P. Sperhake, M. Lütgehetmann, et al., Autopsy findings and venous thromboembolism in patients with COVID-19: a prospective cohort study, Annals of Internal Medicine, (2020), doi: https://doi.org/10.7326/M20-2003w.

[54] M. Madjid, P. Safavi-Naeini, S.D. Solomon, et al., Potential Effects of Coronaviruses on the Cardiovascular System: A Review, JAMA Cardiology, (2020) E1-E10, doi: 10.1001/jamacardio.2020.1286.

[55] J.S.M. Peiris, C.-M. Chu, V.C.-C. Cheng, et al., Clinical progression and viral load in a community outbreak of coronavirus-associated SARS pneumonia: a prospective study, The Lancet, 361 (2003) 1767-1772, doi: 10.1016/S01406736(03)13412-5.

[56] X. Yao, T. Li, Z. He, et al., A pathological report of three COVID-19 cases by minimally invasive autopsies, Zhonghua bing li xue za zhi= Chinese journal of pathology, 49 (2020) E009-E009, doi: 10.3760/cma.j.cn11215120200312-00193

[57] W. Thaweerat, Current evidence on pancreatic involvement in SARS-CoV-2 infection, Pancreatology, (2020), doi.

[58] J.-K. Yang, S.-S. Lin, X.-J. Ji, et al., Binding of SARS coronavirus to its receptor damages islets and causes acute diabetes, Acta diabetologica, 47 (2010) 193-199, doi: 10.1007/s00592-009-0109-4.

[59] S.D. Crowley, S.B. Gurley, M.J. Herrera, et al., Angiotensin II causes hypertension and cardiac hypertrophy through its receptors in the kidney, Proc Natl Acad Sci U S A, 103 (2006) 17985-17990, doi: 10.1073/pnas.0605545103.

[60] N.S. Kalupahana, N. Moustaid-Moussa, The renin-angiotensin system: a link between obesity, inflammation and insulin resistance, Obesity reviews, 13 (2012) 136-149, doi: 10.1111/j.1467-789X.2011.00942.x.

[61] S. Sibley, Hypertension, obesity, and the renin-angiotensin system: a tale of tight associations, Minnesota medicine, 86 (2003) 46-48, doi: https://pubmed.ncbi.nlm.nih.gov/12585560/.

[62] M. Hoffmann, H. Kleine-Weber, S. Schroeder, et al., SARS-CoV-2 cell entry depends on ACE2 and TMPRSS2 and is blocked by a clinically proven protease inhibitor, Cell, 52 (2020) 731-733, doi: https://doi.org/10.1016/j.cell.2020.02.052.

[63] K. Kuba, Y. Imai, S. Rao, et al., A crucial role of angiotensin converting enzyme 2 (ACE2) in SARS coronavirusinduced lung injury, Nature Medicine, 11 (2005) 875-879, doi: 10.1038/nm1267.

[64] M. Roche, P. Rondeau, N.R. Singh, et al., The antioxidant properties of serum albumin, FEBS letters, 582 (2008) 1783-1787, doi: 10.1016/j.febslet.2008.04.057.

[65] M. Anraku, V.T. Chuang, T. Maruyama, et al., Redox properties of serum albumin, Biochimica et biophysica acta, 1830 (2013) 5465-5472, doi: 10.1016/j.bbagen.2013.04.036.

[66] O. Zirafi, P.C. Hermann, J. Münch, Proteolytic processing of human serum albumin generates EPI-X4, an endogenous antagonist of CXCR4, Journal of leukocyte biology, 99 (2016) 863-868, doi: 10.1189/jlb.2MR1115-521RR. 
[67] S. Arques, P. Ambrosi, Human serum albumin in the clinical syndrome of heart failure, Journal of cardiac failure, 17 (2011) 451-458, doi: 10.1016/j.cardfail.2011.02.010.

[68] L. Shuai, J. Wang, D. Zhao, et al., Integrin $\beta 1$ Promotes Peripheral Entry by Rabies Virus, Journal of virology 94 (2020), doi: 10.1128/jvi.01819-19.

[69] W.J. Gan, O.H. Do, L. Cottle, et al., Local Integrin Activation in Pancreatic $\beta$ Cells Targets Insulin Secretion to the Vasculature, Cell reports, 24 (2018) 2819-2826.e2813, doi: 10.1016/j.celrep.2018.08.035.

[70] N. Li, H. Zhou, H. Wu, et al., STING-IRF3 contributes to lipopolysaccharide-induced cardiac dysfunction, inflammation, apoptosis and pyroptosis by activating NLRP3, Redox biology, 24 (2019) 101215, doi: 10.1016/j.redox.2019.101215.

[71] P.M. Becher, S. Hinrichs, N. Fluschnik, et al., Role of Toll-like receptors and interferon regulatory factors in different experimental heart failure models of diverse etiology: IRF7 as novel cardiovascular stress-inducible factor, PLoS One, 13 (2018) e0193844, doi: 10.1371/journal.pone.0193844.

[72] X.A. Wang, R. Zhang, Z.G. She, et al., Interferon regulatory factor 3 constrains IKK $\beta / N F-\kappa B$ signaling to alleviate hepatic steatosis and insulin resistance, Hepatology (Baltimore, Md.), 59 (2014) 870-885, doi: 10.1002/hep.26751.

[73] M. Kumari, X. Wang, L. Lantier, et al., IRF3 promotes adipose inflammation and insulin resistance and represses browning, J Clin Invest, 126 (2016) 2839-2854, doi: 10.1172/JCI86080.

[74] O. Pacha, M.A. Sallman, S.E. Evans, COVID-19: a case for inhibiting IL-17?, (2020) 1-2, doi: 10.1038/s41577-0200328-z.

[75] L.A. Zúñiga, W.-J. Shen, B. Joyce-Shaikh, et al., IL-17 regulates adipogenesis, glucose homeostasis, and obesity, Journal of immunology (Baltimore, Md. : 1950), 185 (2010) 6947-6959, doi: 10.4049/jimmunol.1001269.

[76] C. Sandip, L. Tan, J. Huang, et al., Common variants in IL-17A/IL-17RA axis contribute to predisposition to and progression of congestive heart failure, Medicine, 95 (2016) e4105, doi: 10.1097/md.0000000000004105.

[77] T. Shen, X. Chen, Y. Li, et al., Interleukin-17A exacerbates high-fat diet-induced hepatic steatosis by inhibiting fatty acid $\beta$-oxidation, Biochimica et Biophysica Acta (BBA) - Molecular Basis of Disease, 1863 (2017) 1510-1518, doi: https://doi.org/10.1016/j.bbadis.2017.01.027.

[78] Y.J. Pan, X. Ren, Y.Y. Zhang, et al., IL-17A-mediated ERK1/2/p65 signaling pathway is associated with cell apoptosis after non-alcoholic steatohepatitis, 71 (2019) 302-309, doi: 10.1002/iub.1960.

[79] T. Kisseleva, Does interleukin-17 play the villain in nonalcoholic steatohepatitis?, Hepatology (Baltimore, Md.), 59 (2014) 1671-1672, doi: 10.1002/hep.26955.

[80] F.C. Paquissi, Immunity and Fibrogenesis: The Role of Th17/IL-17 Axis in HBV and HCV-induced Chronic Hepatitis and Progression to Cirrhosis, Front Immunol, 8 (2017) 1195-1195, doi: 10.3389/fimmu.2017.01195.

[81] S. Westhaus, M. Deest, A.T.X. Nguyen, et al., Scavenger receptor class B member 1 (SCARB1) variants modulate hepatitis C virus replication cycle and viral load, J Hepatol, 67 (2017) 237-245, doi: 10.1016/j.jhep.2017.03.020.

[82] S. Samadi, Z. Farjami, Z.S. Hosseini, et al., Rare P376L variant in the SR-BI gene associates with HDL dysfunction and risk of cardiovascular disease, Clinical biochemistry, 73 (2019) 44-49, doi: 10.1016/j.clinbiochem.2019.06.014. 
[83] J. Rejeb, A. Omezzine, I. Boumaiza, et al., Association of three polymorphisms of scavenger receptor class BI gene (exon8, exon1, intron5) with coronary stenosis in a coronary Tunisian population, Gene, 511 (2012) 383-388, doi: 10.1016/j.gene.2012.09.070.

[84] C.K. Roberts, K. Liang, R.J. Barnard, et al., HMG-CoA reductase, cholesterol 7alpha-hydroxylase, LDL receptor, SRB1, and ACAT in diet-induced syndrome X, Kidney international 66 (2004) 1503-1511, doi: 10.1111/j.15231755.2004.00914.x.

[85] V. Malikov, M.H. Naghavi, Localized Phosphorylation of a Kinesin-1 Adaptor by a Capsid-Associated Kinase Regulates HIV-1 Motility and Uncoating, Cell reports, 20 (2017) 2792-2799, doi: 10.1016/j.celrep.2017.08.076.

[86] G. Xu, Y. Ge, X. Tao, et al., MARK2 inhibits the growth of HeLa cells through AMPK and reverses epithelialmesenchymal transition, Oncology reports, 38 (2017) 237-244, doi: 10.3892/or.2017.5686.

[87] J.B. Hurov, M. Huang, L.S. White, et al., Loss of the Par-1b/MARK2 polarity kinase leads to increased metabolic rate, decreased adiposity, and insulin hypersensitivity $<\mathrm{em}>$ in vivo $</ \mathrm{em}>$, Proceedings of the National Academy of Sciences, 104 (2007) 5680-5685, doi: 10.1073/pnas.0701179104.

[88] J.A. Espinoza, P.A. González, A.M. Kalergis, Modulation of Antiviral Immunity by Heme Oxygenase-1, The American journal of pathology, 187 (2017) 487-493, doi: 10.1016/j.ajpath.2016.11.011.

[89] G.S. Drummond, J. Baum, M. Greenberg, et al., HO-1 overexpression and underexpression: Clinical implications, Archives of biochemistry and biophysics, 673 (2019) 108073, doi: 10.1016/j.abb.2019.108073.

[90] M.A. Beck, R.S. Esworthy, Y.S. Ho, et al., Glutathione peroxidase protects mice from viral-induced myocarditis, FASEB journal, 12 (1998) 1143-1149, doi: 10.1096/fasebj.12.12.1143.

[91] S. Yatmaz, H.J. Seow, R.C. Gualano, et al., Glutathione peroxidase-1 reduces influenza A virus-induced lung inflammation, Am J Respir Cell Mol Biol, 48 (2013) 17-26, doi: 10.1165/rcmb.2011-0345OC.

[92] V.C. Sousa, R.F. Carmo, L.R. Vasconcelos, et al., Association of Catalase and Glutathione Peroxidase 1 Polymorphisms with Chronic Hepatitis C Outcome, Annals of human genetics, 80 (2016) 145-153, doi: 10.1111/ahg.12152.

[93] S. Moossavi, S. Besharat, M. Sharafkhah, et al., Inverse Association of Plasma Level of Glutathione Peroxidase with Liver Fibrosis in Chronic Hepatitis B: Potential Role of Iron, Middle East J Dig Dis, 8 (2016) 122-130, doi: 10.15171/mejdd.2016.17.

[94] L. Vávrová, J. Kodydková, M. Zeman, et al., Altered activities of antioxidant enzymes in patients with metabolic syndrome, Obesity facts, 6 (2013) 39-47, doi: 10.1159/000348569.

[95] F.P. Bellinger, A.V. Raman, M.A. Reeves, et al., Regulation and function of selenoproteins in human disease, Biochem J, 422 (2009) 11-22, doi: 10.1042/BJ20090219.

[96] B.L. Grayson, L. Wang, T.M. Aune, Peripheral blood gene expression profiles in metabolic syndrome, coronary artery disease and type 2 diabetes, Genes \& Immunity, 12 (2011) 341-351, doi: 10.1038/gene.2011.13.

[97] Y.-J. Tan, B.C. Fielding, P.-Y. Goh, et al., Overexpression of 7a, a protein specifically encoded by the severe acute respiratory syndrome coronavirus, induces apoptosis via a caspase-dependent pathway, Journal of virology, 78 (2004) 14043-14047, doi: 10.1128/JVI.78.24.14043-14047.2004. 
[98] N. Chen, M. Zhou, X. Dong, et al., Epidemiological and clinical characteristics of 99 cases of 2019 novel coronavirus pneumonia in Wuhan, China: a descriptive study, The Lancet, 395 (2020) 507-513, doi: 10.1016/S0140-6736(20)30211-7. [99] C. Zhang, L. Shi, F.-S. Wang, Liver injury in COVID-19: management and challenges, The lancet Gastroenterology \& hepatology, 5 (2020) 428-430, doi: 10.1016/S2468-1253(20)30057-1. 